\title{
Systematics of jets using multi-wavelength data
}

\author{
Prajval Shastri ${ }^{1,2}$, Estrella Jimenez Gomez ${ }^{1}$, Maitrayee Gupta ${ }^{1}$ \\ and Grzegorz Madejski ${ }^{2}$ \\ ${ }^{1}$ Indian Institute of Astrophysics, Sarjapur Road, Bangalore 560034, India \\ email: prajval.shastri@gmail.com \\ ${ }^{2}$ Kavli Institute of Particle Astrophysics \& Cosmology, Stanford University, Palo Alto CA USA
}

\begin{abstract}
We investigate the systematics of the properties of highly relativistic jets at multiple frequencies, including Fermi and MOJAVE data. We test the hypothesis that the blazar divide constitutes a dichotomy. We also explore possible measures of the Doppler factor for these highly Doppler-beamed active galactic nuclei.
\end{abstract}

Keywords. galaxies :active, galaxies: jets

\section{Introduction}

Most if not all accreting supermassive black holes appear to produce bipolar outflows of hot gas and bipolar synchrotron-emitting plasma jets. In most active galactic nuclei (AGN) these jets appear quenched well within their host galaxies, and they are referred to as "radio-quiet" AGN in the literature. Their jets appear not to be significantly relativistic (e.g., Lal et al. 2011; Middelberg et al. 2004) except in the case of the Narrow-Line Seyfert 1 type AGN (e.g., J. Richards, these proceedings). In a small minority (15-20\%) of the AGN, however, the jets appear to be launched with large kinetic power and at bulk relativistic speeds, and these jets reach scales of several hundred kiloparsecs and even a megaparsec. AGN with such jets are referred to as "radio-loud". It has been well-established that when such jets are oriented close to our line of sight, relativistic aberration strongly affects their observed parameters (e.g., Blandford \& Konigl 1979) and their emission is heavily Doppler beamed. These beamed jets dominate surveys at gamma-ray energies (e.g., Ackermann et al. 2011), and have also been the primary candidates for the measurement of the apparent proper motion of the jet components via Very Long Baseline Interferometry (e.g., the MOJAVE project: Lister et al. 2009). We present here some work in progress that investigates the systematics of highly relativistically beamed jets, primarily using Fermi and MOJAVE data.

\section{The blazar divide}

Blazars are AGN that exhibit large variability and polarization of their contnuum emission at virtually all wavelengths observed. These characteristics are widely recognised as being hallmarks of relativistic beaming of the large kinetic power jets when the jets are close to the line-of-sight. Several quasars, which, by definition, have prominent broad emission lines in their optical and IR spectra, show blazar characterisitcs and being quasars, their radio structures are of the Fanaroff-Riley II type. There are also AGN which exhibit blazar characteristics, but have only weak or no emission lines in their optical spectra. Referred to as BL Lacertids or BL Lacs in the literature, they are believed to have the synchrotron component overwhelmingly dominate the emission at all wavelengths 

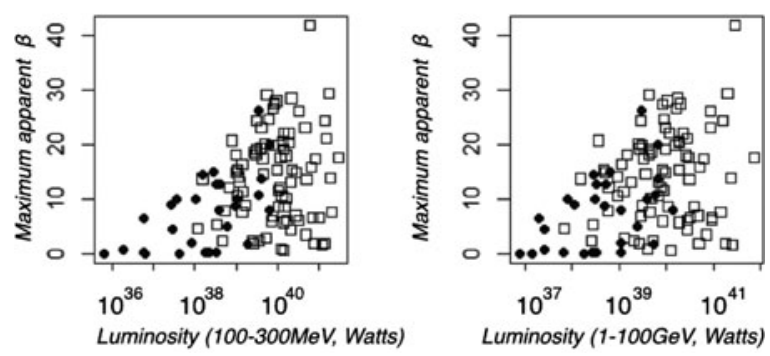

Figure 1. Plot of $\beta_{m a x}^{a p p}$ versus gamma-ray luminosity for the Fermi blazars that also have MOJAVE data (Lister et al. 2009). Plots for the Fermi $100-300 \mathrm{MeV}$ and 1-100 GeV bands are shown. Plots for the other Fermi bands are similar. Although there is an overall trend for the blazars in that the gamma-ray luminosity correlates with the $\beta_{\text {max }}^{a p p}$, this is mostly due to the systematic difference in gamma-ray luminosity between the BL Lacs and quasars.

including any emission lines. Their extended radio structures are not as well-determined, and are found to be of both Fanaroff-Riley types or even mixed (e.g., Kharb et al. 2010).

The physical reason for this blazar divide is not clear. In other AGN sub-classes that are otherwise similar, the presence of obscuring toroidal material is invoked to explain the absence of observed emission-line regions (e.g., Antonucci 1993). This explanation clearly cannot be invoked to explain the blazar divide, however, since all blazar jets are Doppler-beamed and are therefore pointed close to the line of sight.

Ghisellini et al. (2009) have argued that the divide is not a dichotomy but a continuity, with a physical origin. They suggest that the separation between the BL Lacs and quasars is a result of a critical accretion rate, below which the accretion is radiatively inefficient, resulting in weak or no broad emission lines as in the BL Lacs. The gamma-ray photons emerge from a photo-starved environment in these cases and therefore have a harder spectrum. Above the critical accretion rate, not only would the broad emission lines be visible, the inverse-Compton emission would be primarily external Compton, and with a relatively softer the gamma-ray spectrum. Ghisellini et al. (2009) further suggest that this interpretation is also consistent with unification that posits that the Fanaroff-Riley radio galaxies of types I and II constitute the parent population of the beamed BL Lacs and quasars respectively. The results of Kharb et al. (2010) call the latter interpretation into question, however.

\section{Systematics}

We explore the connections between the gamma-ray properties and jet kinematics. The MOJAVE project (Lister et al. 2009) has resulted in the measurement of the apparent proper motion speeds of the pc-scale jet components of a large sample of blazars, which, not surprisingly, have significant overlap with the Fermi AGN sample.

In Fig. 1 the maximum measured apparent proper motion speed $\left(\beta_{\text {max }}^{a p p}\right)$ of the pc-scale jet component over the time-baseline of the MOJAVE observations (MOJAVE data: Lister et al. (2009)) is plotted against the gamma-ray luminosity measured by Fermi. The sample of objects are the AGNs from the combined 1LAC (Abdo et al. 2010) and 2LAC (Ackermann et al. 2011) AGN catalogues which also have MOJAVE data (Lister et al. 2009). On the face of it, blazars with higher $\beta_{\text {max }}^{a p p}$ appear to have larger gamma-ray luminosities. This trend is not at all surprising, given that the gamma-ray emission is expected to be heavily Doppler-beamed, and larger the beaming, higher the expected $\beta_{\text {max }}^{a p p}$, except at nearly parallel lines of sight to the accretion axis. When the blazars are disaggregated by sub-type, however, the apparent systematic trend can be clearly seen 

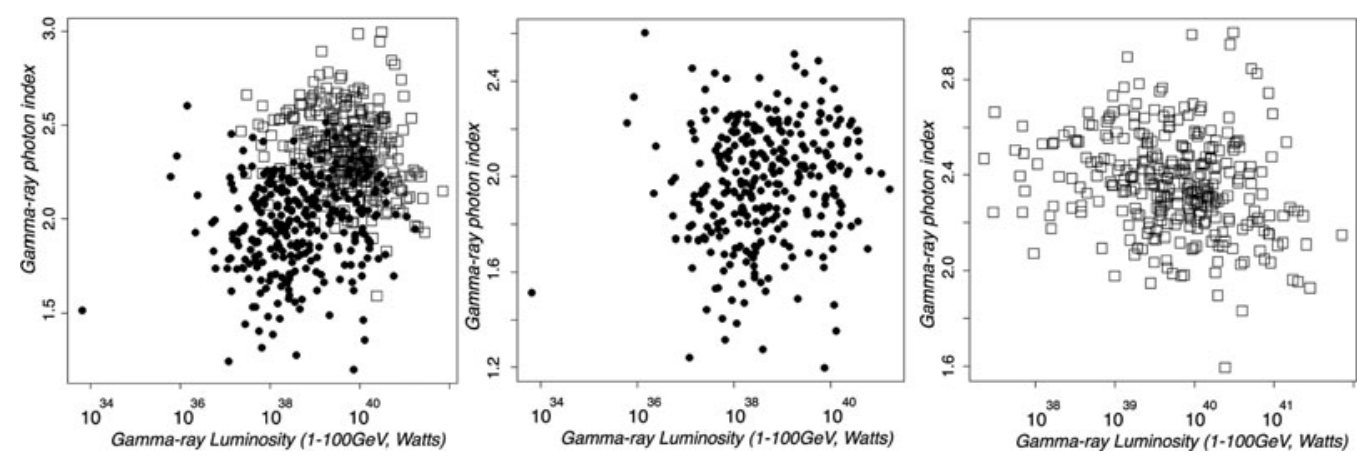

Figure 2. Plot of the gamma-ray photon index versus the gamma-ray luminosity for the BL Lacs and Quasars. In the middle and right panels, the BL Lacs and quasars respectively are plotted separately (cf., also Ghisellini et al. (2009); Ackermann et al. (2011) and Lott et al. in these proceedings).

as largely due to the systematic difference in the gamma-ray luminosity between the BL Lacs and quasars, i.e., the Malmquist bias, and the trend within each sub-class is much less significant.

Fig. 2 shows the sample AGN in the luminosity-spectral index plane in gamma-rays. The plot is broadly consistent with the earlier conclusions of Ghisellini et al. (2009) and Ackermann et al. (2011) that BL Lacs and quasars occupy different regions of this plane. Ghisellini et al. (2009) suggested, however, that there is a continuity between the two blazar subclasses.

When the BL Lacs and quasars are considered separately (Fig. 2, right panel), they clearly show qualitatively different trends of the photon index with gamma-ray luminosity (as indeed was suggested by Ackermann et al. (2011).) While it may still be true that the accretion in BL Lacs is less radiatively efficient, the data are clearly not consistent with a continuity of properties between BL Lacs and beamed quasars.

In order to investigate these relationships further, it is important to have a handle on the Doppler factor, since relativistic aberration severely affects the observed properties. The radio core-dominance, i.e., the ratio of the nuclear to lobe radio emission in the AGN rest frame (Kapahi \& Saikia 1982; Wills et al. 1992) has been shown to be a robust statistical measure of orientation of the radio jet axis to the line-of-sight.

For highly Doppler-beamed AGN such as the blazars under consideration here, however, the core-dominance parameter will work well only if the nuclear emission and extended (or total) radio emission are measured simultaneously. Blazars are both highly core-dominant and highly variable. Radio images that are of high enough resolution to delineate the nucleus (e.g., Kharb et al. 2010, VLA-A array measurements), typically miss a significant fraction of the extended flux density. The total flux density can be used instead but only if measured at a close enough epoch, because otherwise erroneous values will result due to the high degree of variability. Simultaneous high-resolution nuclear emission measurements and total flux density measurements are not available for the sample at hand.

Instead, we explore an alternative measure obtained at optical wavelengths. Nilsson et al. (2003) have imaged the host galaxies of a sample of BL Lacs with good seeing using the Nordic Optical Telescope. The nuclear optical emission of BL Lacs is primarily synchrotron emission from the nuclear jet and is Doppler beamed. The host galaxy emission is clearly unbeamed. The ratio of the nucleus to host-galaxy emission can therefore be used as a statistical proxy for the angle to the line of sight. Fig. 3 shows plots of the 

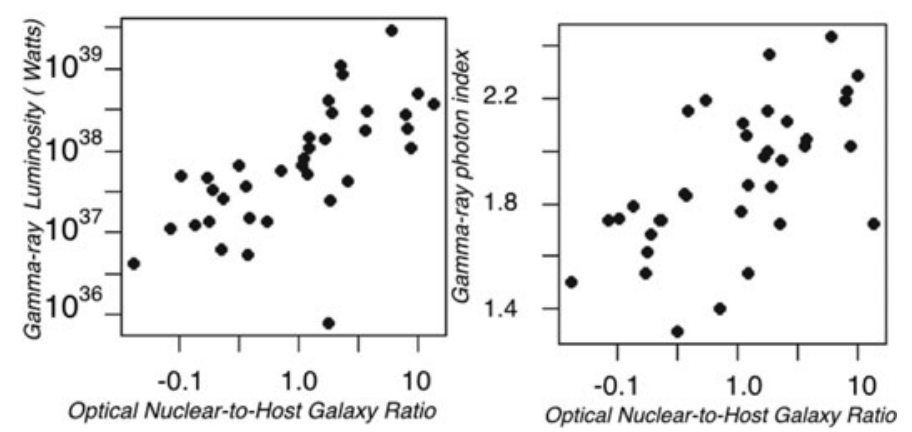

Figure 3. Plot of the gamma-ray luminosity in the $1-100 \mathrm{GeV}$ band (left) and gamma-ray photon index versus the optical nuclear-to-host galaxy ratio.

gamma-ray luminosity and gamma-ray photon index versus the optical nuclear to host galaxy ratio. The gamma-ray emission which is expected to be highly Doppler-beamed, significantly correlates with this parameter. The plot also shows that the gamma-ray photon index appears to soften with larger Doppler factors.

\section{Conclusions}

- The analysis presented here shows that the data are qualitatively consistent with the hypothesis that BL Lac jets are launched in a relatively photon-starved environment.

- The data suggest that the blazar-divide is indeed a dichotomy.

- Quantitative modeling, and exploring whether the blazar divide reflects the FanaroffRiley divide requires accounting for Malmquist bias, and selection effects.

- Quantitative modeling also requires a robust proxy for the inclination of the jet to the line of sight. The standard statistical orientation measure, viz., the radio coredominance parameter, has limited reliability within the blazar class because of missing extended flux density in most measurements and also because of variability.

- The optical nuclear-to-host galaxy ratio may be an alternative proxy for the angle of inclination of the jet to the line of sight.

- The larger number of AGN available from the third Fermi catalogue 3FGL (e.g., Lott, these proceedings) may allow accounting for the Malmquist bias in blazar samples.

\section{Acknowledgements}

This research has made use of data from the Fermi LAT public archive and from the MOJAVE database that is maintained by the MOJAVE team Lister et al. (2009); data and/or software provided by the High Energy Astrophysics Science Archive Research Center (HEASARC), which is a service of the Astrophysics Science Division at NASA/GSFC and the High Energy Astrophysics Division of the Smithsonian Astrophysical Observatory; and the NASA/IPAC Extragalactic Database (NED), which is operated by the Jet Propulsion Laboratory, California Institute of Technology, under contract with the National Aeronautics and Space Administration. PS acknowledges the support from the Fulbright-Nehru Fellowship. EJG acknowledges support from the FARO 
Global grants programme, Spain. MG acknowledges support from the student internship programme of the Indian Institute of Astrophysics.

\section{References}

Abdo, A. A., Ackermann, M., Ajello, M., et al. 2010, ApJ, 715, 429

Ackermann, M., Ajello, M., Allafort, A., et al. 2011, ApJ, 743, 171

Antonucci, R. 1993, ARAA, 31, 473

Blandford, R. D. \& Konigl, A. 1979, ApJ, 232, 34

Ghisellini, G., Maraschi, L., \& Tavecchio, F. 2009, MNRAS, 396, L105

Kapahi, V. K. \& Saikia, D. J. 1982, JA\&A, 3, 465

Kharb, P., Lister, M. L., \& Cooper, N. J. 2010, ApJ, 710, 764

Lal, D. V., Shastri, P., \& Gabuzda, D. C. 2011, ApJ, 731, 68

Lister, M. L., Aller, H. D., Aller, M. F., et al. 2009, AJ, 137, 3718

Middelberg, E., Roy, A. L., Nagar, N. M., et al. 2004, A\& A, 417, 925

Nilsson, K., Pursimo, T., Heidt, J., et al. 2003, A\&A, 400, 95

Wills, B. J., Wills, D., Breger, M., Antonucci, R. R. J., \& Barvainis, R. 1992, ApJ, 398, 454 